\title{
ON NON-HOMOGENEOUS CANONICAL THIRD-ORDER LINEAR DIFFERENTIAL EQUATIONS
}

\author{
N. PARHI \\ (Received 10 January 1991; revised 8 August 1991) \\ Communicated by A. J. Pryde
}

\begin{abstract}
In this paper sufficient conditions have been obtained for non-oscillation of non-homogeneous canonical linear differential equations of third order. Some of these results have been extended to non-linear equations.
\end{abstract}

1991 Mathematics subject classification (Amer. Math. Soc.): $34 \mathrm{C} 10,34 \mathrm{C} 11$.

\section{Introduction}

In [1] Barrett considered homogeneous third-order linear differential equations of the form

$$
\left[r_{2}(t)\left\{\left(r_{1}(t) y^{\prime}\right)^{\prime}+q_{1}(t) y\right\}\right]^{\prime}+q_{2}(t)\left(r_{1}(t) y^{\prime}\right)=0
$$

where $r_{1}, r_{2}, q_{1}$ and $q_{2} \in C([a, \infty), R), a \in R, r_{1}(t)>0$ and $r_{2}(t)>0$. By a solution of $(\mathrm{H})$ on $[a, \infty)$ we mean a function $y \in C^{1}([a, \infty), R)$ such that $r_{1} y^{\prime}$ and $r_{2}\left\{\left(r_{1} y^{\prime}\right)^{\prime}+q_{1} y\right\} \in C^{1}([a, \infty), R)$ and $(\mathrm{H})$ is satisfied identically. We call $(\mathrm{H})$ the third-order canonical form. The adjoint of $(\mathrm{H})$ is given by

$$
\left[r_{1}(t)\left\{\left(r_{2}(t) y^{\prime}\right)^{\prime}+q_{2}(t) y\right\}\right]^{\prime}+q_{1}(t)\left(r_{2}(t) y^{\prime}\right)=0 .
$$

We may note that $\left(\mathrm{H}^{*}\right)$ is obtained from $(\mathrm{H})$ by interchanging $r_{1}$ with $r_{2}$ and $q_{1}$ with $q_{2}$. The non-homogeneous equations associated with $(\mathrm{H})$ and $\left(\mathrm{H}^{*}\right)$ are given, respectively, by (NH)

$$
\left[r_{2}(t)\left\{\left(r_{1}(t) y^{\prime}\right)^{\prime}+q_{1}(t) y\right\}\right]^{\prime}+q_{2}(t)\left(r_{1}(t) y^{\prime}\right)=f_{1}(t)
$$

and $\left(\mathrm{NH}^{*}\right)$

$$
\left[r_{1}(t)\left\{\left(r_{2}(t) y^{\prime}\right)^{\prime}+q_{2}(t) y\right\}\right]^{\prime}+q_{1}(t)\left(r_{2}(t) y^{\prime}\right)=g_{1}(t)
$$

(C) 1994 Australian Mathematical Society 0263-6115/94\$A2.00+0.00 
with $f_{1}$ and $g_{1} \in C([a, \infty), R)$ such that $f_{1}(t) \geq 0$ and $g_{1}(t) \geq 0$.

Suppose that $\int_{a}^{\infty} d t / r_{1}(t)=\infty$. The Liouville transformation $s=R(t), x(s)=$ $y(t)$, where $R(t)=\int_{a}^{t} d u / r_{1}(u)$, transforms (NH) into

$$
\frac{d}{d s}\left[\frac{r_{2}(t)}{r_{1}(t)} \frac{d^{2} x}{d s^{2}}+r_{2}(t) q_{1}(t) x\right]+r_{1}(t) q_{2}(t) \frac{d x}{d s}=r_{1}(t) f_{1}(t)
$$

with $t=R^{-1}(s)$. If $\int_{a}^{\infty} d t / r_{1}(t)<\infty$, then the Kummer transformation $s=1 / \rho(t)$, $x(s)=s y(t)$, where $\rho(t)=\int_{t}^{\infty} d u / r_{1}(u)$, transforms (NH) into

$$
\frac{d}{d s}\left[\frac{r_{2}(t)}{r_{1}(t)} s^{3} \frac{d^{2} x}{d s^{2}}+\frac{r_{2}(t)}{s} q_{1}(t) x\right]+\frac{r_{1}(t) q_{2}(t)}{s} \frac{d x}{d s}-\frac{r_{1}(t) q_{2}(t)}{s^{2}} x=\frac{r_{1}(t)}{s^{2}} f_{1}(t)
$$

with $t=\rho^{-1}(1 / s)$. However, Equation (2) may be written as

(3) $\frac{d}{d s}\left[\sigma(s) \frac{d^{2} x}{d s^{2}}+\left(\lambda(s)-\int_{a}^{s} v(u) d u\right) x\right]+\left[\mu(s)+\int_{a}^{s} v(u) d u\right] \frac{d x}{d s}=\frac{r_{1}(t)}{s^{2}} f_{1}(t)$ where $\sigma(s)=r_{2}(t) s^{3} / r_{1}(t), \lambda(s)=r_{2}(t) q_{1}(t) / s, \mu(s)=r_{1}(t) q_{2}(t) / s$ and $\nu(s)=$ $r_{1}(t) q_{2}(t) / s^{2}$.

We may note that $x(s)$ is non-oscillatory if and only if $y(t)$ is non-oscillatory. Furthermore, Equations (1) and (3) have the same general form. If $\int_{a}^{\infty} d t / r_{2}(t)=\infty$ or $\int_{a}^{\infty} d t / r_{2}(t)<\infty$, then $\left(\mathrm{NH}^{*}\right)$ is transformed into an equation of the type (1) or (3) which is obtained by interchanging $r_{1}$ with $r_{2}$ and $q_{1}$ with $q_{2}$. Hence it is enough to study the equations of the form

$$
\left(r(t) y^{\prime \prime}+p(t) y\right)^{\prime}+q(t) y^{\prime}=f(t)
$$

where $p, q, r$ and $f \in C([a, \infty), R), r(t)>0$ and $f(t) \geq 0$.

We recall that a function $y \in C([a, \infty), R)$ is said to be oscillatory if for every $t_{1} \geq a$ there exist $t_{2}$ and $t_{3}\left(t_{1}<t_{2}<t_{3}\right)$ such that $y\left(t_{2}\right)>0$ and $y\left(t_{3}\right)<0$. It is said to be of $Z$-type if it has arbitrarily large zeros but is ultimately non-negative or non-positive. A function $y(t)$ is said to be non-oscillatory if it is neither oscillatory nor of Z-type. Equation (E) is said to be non-oscillatory if all of its solutions are non-oscillatory.

Linear non-homogeneous third order differential equations of the type

$$
\left(r(t) y^{\prime \prime}\right)^{\prime}+q(t) y^{\prime}+p(t) y=f(t)
$$

occur in the study of the entry flow phenomenon in hydrodymics [3]. We note that Equation (4) is a particular case of (E). Indeed, we may write Equation (4) as

$$
\left[r(t) y^{\prime \prime}+\left(\int_{a}^{t} p(s) d s\right) y\right]^{\prime}+\left(q(t)-\int_{a}^{t} p(s) d s\right) y^{\prime}=f(t) .
$$


Unlike the second order case, equation (4) cannot be transformed to an equation of the type

$$
x^{\prime \prime \prime}+c(t) x^{\prime}+b(t) x=h(t)
$$

when $\int_{a}^{\infty} d t / r(t)=\infty$ or $\int_{a}^{\infty} d t / r(t)<\infty$.

The purpose of this paper is to study non-oscillatory behaviour of solutions of $(E)$. In the process, we obtain a result which generalizes a result in [5]. In Section 2 we obtain sufficient conditions for non-oscillation of $(E)$. It is interesting to note that this study is applicable to a class of non-linear equations. Section 3 deals with the relation between three independent solutions of $(E)$.

\section{Non-oscillatory behaviour of solutions}

In this section we obtain sufficient conditions for non-oscillation of (E). The same techniques are then used to obtain non-oscillation results for certain classes of nonlinear equations (see Equations (7) - (11) below).

THEOREM 1. If $p(t) \leq 0$ and $q(t) \leq 0$ for large $t$, then $(\mathrm{E})$ is non-oscillatory.

PROOF. Let $y(t)$ be a solution of (E) on $[a, \infty)$. Let $p(t) \leq 0$ and $q(t) \leq 0$ for $t \geq t_{0} \geq a$. Let $y(t)$ be of non-negative Z-type with consecutive double zeros at $t_{1}$ and $t_{2}\left(t_{0}<t_{1}<t_{2}\right)$. So there exists a $b \in\left(t_{l}, t_{2}\right)$ such that $y^{\prime}(b)=0, y^{\prime \prime}(b) \leq 0$ and $y^{\prime}(t)>0$ for $t \in\left(t_{1}, b\right)$. Integrating (E) from $t_{1}$ to $b$, we get

$$
\begin{aligned}
0 & \geq r(b) y^{\prime \prime}(b)+p(b) y(b)-c\left(t_{1}\right) y^{\prime \prime}\left(t_{1}\right) \\
& =\int_{t_{1}}^{b} f(t) d t-\int_{t_{1}}^{b} q(t) y^{\prime}(t) d t>0
\end{aligned}
$$

because $y^{\prime \prime}\left(t_{1}\right) \geq 0$. Suppose that $\mathrm{y}(\mathrm{t})$ is a non-positive Z-type solution with consecutive double zeros at $t_{1}$ and $t_{2}\left(t_{0}<t_{1}<t_{2}\right)$. Then there exists $b \in\left(t_{1}, t_{2}\right)$ such that $y^{\prime}(b)=0$ and $y^{\prime}(t)>0$ for $t \in\left(b, t_{2}\right)$. We note that $y^{\prime \prime}(b) \geq 0$ and $y^{\prime \prime}\left(t_{2}\right) \leq 0$. Now integrating (E) from $b$ to $t_{2}$ yields

$$
\begin{aligned}
0 & \geq r\left(t_{2}\right) y^{\prime \prime}\left(t_{2}\right)+r(b) y^{\prime \prime}(b)-p(b) y(b) \\
& =\int_{b}^{t_{2}} f(t) d t-\int_{b}^{t_{2}} q(t) y^{\prime}(t) d t>0
\end{aligned}
$$

a contradiction. Hence $y(t)$ cannot be of Z-type.

Suppose that $y(t)$ is an oscillatory solution with consecutive zeros at $t_{1}, t_{2}$ and $t_{3}$ $\left(t_{0}<t_{1}<t_{2}<t_{3}\right)$ such that $y(t)<0$ for $t \in\left(t_{1}, t_{2}\right)$ and $y(t)>0$ for $t \in\left(t_{2}, t_{3}\right)$. So there exist $b \in\left(t_{1}, t_{2}\right)$ and $c \in\left(t_{2}, t_{3}\right)$ such that $y^{\prime}(b)=0, y^{\prime}(c)=0, y^{\prime}(t)>0$ for 
$t \in\left(b, t_{2}\right)$ and $y^{\prime}(t)>0$ for $t \in\left(t_{2}, c\right)$. If $y^{\prime \prime}\left(t_{2}\right) \geq 0$, then integrating (E) from $t_{2}$ to $c$, we obtain

$$
0 \geq r(c) y^{\prime \prime}(c)+p(c) y(c)-r\left(t_{2}\right) y^{\prime \prime}\left(t_{2}\right)=\int_{t_{2}}^{c} f(t) d t-\int_{t_{2}}^{c} q(t) y^{\prime}(t) d t>0,
$$

a contradiction because $y^{\prime \prime}(c) \leq 0$. Furthermore, if $y^{\prime \prime}\left(t_{2}\right)<0$ then integrating (E) from $b$ to $t_{2}$ yields

$$
0>r\left(t_{2}\right) y^{\prime \prime}\left(t_{2}\right)-r(b) y^{\prime \prime}(b)-p(b) y(b)=\int_{b}^{t_{2}} f(t) d t-\int_{b}^{t_{2}} q(t) y^{\prime}(t) d t>0,
$$

a contradiction, because $y^{\prime \prime}(b) \geq 0$. Hence $y(t)$ cannot be oscillatory. This completes the proof of the theorem.

THEOREM $1^{\prime}$. If $\int_{a}^{t} p(\theta) d \theta \leq 0$ and $q(t) \leq \int_{a}^{t} p(\theta) d \theta$ for large $t$, then Equation (4) is non-oscillatory.

PROOF. This result follows from Theorem 1.

REMARK. We note that $p(t) \leq 0$ implies $\int_{a}^{t} p(\theta) d \theta \leq 0$ but the converse is not necessarily true. Furthermore, $p(t)-q^{\prime}(t) \geq 0$ implies $q(t) \leq \int_{a}^{t} p(\theta) d \theta$, if $q(a) \leq 0$. Hence Theorem $1^{\prime}$ improves Theorem 2.1 in [5].

THEOREM 2. If $p(t) \geq 0, q(t) \leq 0$ and $p(s)+q(t) \leq 0$, for $t$ and $s \in[a, \infty)$ and $p(s)+q(t) \not \equiv 0$ on any subinterval of $[a, \infty)$, then $(\mathrm{E})$ is non-oscillatory.

PROOF. Let $y(t)$ be a solution of (E) on $[a, \infty)$. If $y(t)$ is of non-negative Z-type with consecutive double zeros at $t_{1}$ and $t_{2}\left(a<t_{1}<t_{2}\right)$, then there exists a point $b \in\left(t_{1}, t_{2}\right)$ such that $y^{\prime}(b)=0$ and $y^{\prime}(t)>0$ for $t \in\left(t_{1}, b\right)$. Since $y^{\prime \prime} \geq 0$ and $y^{\prime \prime}(b) \leq 0$, then integrating (E) from $t_{1}$ to $b$, we obtain

$$
\begin{aligned}
0 & \geq r(b) y^{\prime \prime}(b)-r\left(t_{1}\right) y^{\prime \prime}\left(t_{1}\right) \\
& \geq-p(b) y(b)-\int_{t_{1}}^{b} q(t) y^{\prime}(t) d t \\
& \geq-\int_{t_{1}}^{b}[q(t)+p(b)] y^{\prime}(t) d t>0,
\end{aligned}
$$

a contradiction. If $y(t)$ is of non-positive Z-type with consecutive double zeros at $t_{1}$ and $t_{2}\left(a<t_{1}<t_{2}\right)$, then there exists a point $b \in\left(t_{1}, t_{2}\right)$ such that $y^{\prime}(b)=0$ and 
$y^{\prime}(t)>0$ for $t \in\left(b, t_{2}\right)$. Clearly $y^{\prime \prime}(b) \geq 0$ and $y^{\prime \prime}\left(t_{2}\right) \leq 0$. So integrating (E) from $b$ to $t_{2}$ yields

$$
\begin{aligned}
0 & \geq r\left(t_{2}\right) y^{\prime \prime}\left(t_{2}\right)-r(b) y^{\prime \prime}(b) \\
& \geq p(b) y(b)-\int_{b}^{t_{2}} q(t) y^{\prime}(t) d t \\
& \geq-\int_{b}^{t_{2}}[q(t)+p(b)] y^{\prime}(t) d t>0,
\end{aligned}
$$

a contradiction. Hence $y(t)$ cannot be of Z-type.

Suppose that $y(t)$ is oscillatory. Let $t_{1}, t_{2}, t_{3}\left(a<t_{1}<t_{2}<t_{3}\right)$ be consecutive zeros of $y(t)$ such that $y^{\prime}\left(t_{1}\right) \leq 0$ and $y^{\prime}\left(t_{2}\right) \geq 0$ and $y^{\prime}\left(t_{3}\right) \leq 0$. So there exist $b \in\left(t_{1}, t_{2}\right)$ and $c \in\left(t_{2}, t_{3}\right)$ such that $y^{\prime}(t)>0$ for $t \in\left(b, t_{2}\right)$ and $t \in\left(t_{2}, c\right)$. Clearly, $y^{\prime \prime}(b) \geq 0$ and $y^{\prime \prime}(c) \leq 0$. If $y^{\prime \prime}\left(t_{2}\right) \geq 0$, then integrating (E) from $t_{2}$ to $c$, we obtain

$$
\begin{aligned}
0 & \geq r(c) y^{\prime \prime}(c)-r\left(t_{2}\right) y^{\prime \prime}\left(t_{2}\right) \\
& \geq-p(c) y(c)-\int_{t_{2}}^{c} q(t) y^{\prime}(t) d t \\
& \geq-\int_{t_{2}}^{c}[q(t)+p(c)] y^{\prime}(t) d t>0,
\end{aligned}
$$

a contradiction. If $y^{\prime \prime}\left(t_{2}\right)<0$, then integrating (E) from $b$ to $t_{2}$, we get

$$
\begin{aligned}
0 & >r\left(t_{2}\right) y^{\prime \prime}\left(t_{2}\right)-r(b) y^{\prime \prime}(b) \\
& \geq p(b) y(b)-\int_{b}^{t_{2}} q(t) y^{\prime}(t) d t \\
& \geq \int_{b}^{t_{2}}[q(t)+p(b)] y^{\prime}(t) d t>0 .
\end{aligned}
$$

This contradiction completes the proof of the theorem.

REMARK. The condition $p(s)+q(t) \leq 0$ for $t$ and $s \in[a, \infty)$ is equivalent to $p(s) \leq|q(t)|$. Hence $0 \leq p(s) \leq K \leq|q(t)|$ for $t$ and $s \in[a, \infty)$, where $K>0$ is a constant, implies that $p(s)+q(t) \leq 0$.

THEOREM $2^{\prime}$. If $\int_{a}^{t} p(u) d u \geq 0, \quad q(t) \leq \int_{a}^{t} p(u) d u$ and $\int_{a}^{s} p(u) d u \leq$ $\int_{a}^{t} p(u) d u-q(t)$, then Equation (4) is non-oscillatory.

This follows from Theorem 2.

EXAMPLE. Consider

$$
\left(2 t^{3} y^{\prime \prime}+\frac{1}{t+2} y\right)^{\prime}-4 t y^{\prime}=4 t^{2}+\frac{t(t+4)}{(t+2)^{2}}, \quad t \geq 1 .
$$


Clearly $p(s)=1 /(s+2) \leq 1 / 3<4 t=|q(t)|$ for $s, t \geq 1$. From Theorem 2 it follows that Equation (5) is non-oscillatory. In particular, $y(t)=t^{2}$ is a non-oscillatory solution of the equation. Note that Equation (5) may be written as

$$
\left(2 t^{3} y^{\prime \prime}\right)^{\prime}-\left(4 t-\frac{1}{t+2}\right) y^{\prime}-\frac{1}{(t+2)^{2}} y=4 t^{2}+\frac{t(t+4)}{(t+2)^{2}}, \quad t \geq 1 .
$$

Clearly, Theorem 2' cannot be applied to (5). We note that

$$
\int_{1}^{t}-\left[\frac{1}{(u+2)^{2}}\right] d u=\frac{1}{t+2}-\frac{1}{3} .
$$

However Theorems 2 and $2^{\prime}$ can be applied to the equation

$$
\left(5 t^{4} y^{\prime \prime}+2 y\right)^{\prime}-8 y^{\prime}=40 t^{3}-12 t, \quad t \geq 0,
$$

which admits the non-oscillatory solution $y(t)=t^{2}$.

The proofs of the following two results are similar to the proofs of Theorem 2 and $2^{\prime}$ and hence will be omitted.

THEOREM 3. If $p(t) \leq 0, q(t) \geq 0$ and $p(t)+q(s) \leq 0$ for $t$ and $s \in[a, \infty)$ such that $p(t)+q(s) \not \equiv 0$ on any subinterval of $[a, \infty)$, then $(\mathrm{E})$ is non-oscillatory.

THEOREM $3^{\prime}$. If $\int_{a}^{t} p(u) d u \leq 0, \quad q(t) \geq \int_{a}^{t} p(u) d u$ and $\int_{a}^{t} p(u) d u \leq$ $\int_{a}^{s} p(u) d u-q(s)$, then Equation (4) is non-oscillatory.

Our last non-oscillation result for linear equations is contained in the following theorem

THEOREM 4. Let $p(t) \geq 0$ and $q(t) \geq 0$. If $\lim _{t \rightarrow \infty} f(t) /(p(s)+q(t))=\infty$ uniformly for $s \geq a$, then every solution of (E) whose first derivative is bounded is non-oscillatory.

PROOF. Let $y(t)$ be a solution of (E) on $[a, \infty)$ such that $\left|y^{\prime}(t)\right| \leq L$ for $t \geq a$. From the given hypothesis it follows that there exists a $T>a$, independent of $s$, such that $f(t)>L(p(s)+q(t))$ for $t \geq T$.

Suppose that $y(t)$ is of non-negative Z-type with consecutive double zeros at $t_{1}$ and $t_{2}\left(T<t_{1}<t_{2}\right)$. Then there exists $b \in\left(t_{1}, t_{2}\right)$ such that $y^{\prime}(b)=0$ and $y^{\prime}(t)>0$ for $t \in\left(t_{1}, b\right)$. Now integrating (E) from $t_{1}$ to $b$, we get

$$
\begin{aligned}
0 & \geq r(b) y^{\prime \prime}(b)-r\left(t_{1}\right) y^{\prime \prime}\left(t_{1}\right) \\
& =-p(b) y(b)-\int_{t_{1}}^{b} q(t) y^{\prime}(t) d t+\int_{t_{1}}^{b} f(t) d t
\end{aligned}
$$




$$
\begin{aligned}
& =-\int_{t_{1}}^{b}[q(t)+p(b)] y^{\prime}(t) d t+\int_{t_{1}}^{b} f(t) d t \\
& \geq \int_{t_{1}}^{b}[f(t)-L(q(t)+p(b))] d t>0,
\end{aligned}
$$

a contradiction. Similar contradiction may be obtained in case $y(t)$ is non-positive Z-type or oscillatory. Hence the theorem is proved.

REMARK. The Liouville transformation transforms

$$
\left[r_{2}(t)\left(\left(r_{1}(t) y^{\prime}\right)^{\prime}+q_{1}(t) y^{\alpha}\right)\right]^{\prime}+q_{2}(t)\left(r_{1}(t) y^{\prime}\right)^{\beta}=f_{1}(t)
$$

where $q_{1}, q_{2}, r_{1}, r_{2}$ and $f_{1}$ are as in (NH) and each of $\alpha>0$ and $\beta>0$ is a quotient of odd integers, to an equation of the type

$$
\left(r(t) y^{\prime \prime}+p(t) y^{\alpha}\right)^{\prime}+q(t)\left(y^{\prime}\right)^{\beta}=f(t) .
$$

However, the Kummer transformation fails to do so.

THEOREM 5. If $p(t) \leq 0$ and $q(t) \leq 0$, then (7) is non-oscillatory.

The proof of this theorem is similar to that of Theorem 1 and hence is omitted.

REMARK. Theorems $1-5$ all remain true if the condition, ' $f(t) \geq 0$ ' is replaced by ' $f(t) \leq 0$ '.

Equations of the type

$$
y^{\prime \prime \prime}+y y^{\prime \prime}+\lambda\left[1-\left(y^{\prime}\right)^{2}\right]=0
$$

arise in boundary layer theory in fluid Mechanics cite[p. 520]2. The particular case of $(8), y^{\prime \prime \prime}+y y^{\prime \prime}=0$, is known as the Blasius equation. In the following we study the non-oscillatory behaviour of solutions of the non-homogeneous Blasius equation

$$
y^{\prime \prime \prime}+y y^{\prime \prime}=f(t)
$$

where $f \in C([a, \infty), R)$ is such that $f(t) \geq 0$.

THEOREM 6. All solutions of Equation (9) are non-oscillatory.

PROOF. Equation (9) may be written as

$$
\left[y^{\prime \prime}+y y^{\prime}\right]^{\prime}=\left(y^{\prime}\right)^{2}+f(t) .
$$

Let $y(t)$ be a solution of $(10)$ on $[a, \infty)$. Proceeding exactly as in Theorem 1 , one may show that $y(t)$ cannot be of Z-type or oscillatory. Hence $y(t)$ is non-oscillatory. 
The following examples illustrate the theorem.

\section{EXAMPLES.}

(i) The equation $y^{\prime \prime \prime}+y y^{\prime \prime}=0$ admits both positive and negative solutions $y_{1}(t)=t$ and $y_{2}(t)=-t$,

(ii) The equation $y^{\prime \prime \prime}+y y^{\prime \prime}=8 / t^{4}, t \geq 1$, admits the positive bounded solution $y(t)=4 / t$

(iii) $y(t)=-e^{-t}$ is a bounded negative solution of

$$
y^{\prime \prime \prime}+y y^{\prime \prime}=e^{-t}+e^{-2 t}, \quad t \geq 0 .
$$

The asymptotic behaviour of solutions of Equation (8) has been studied by Hartman [2]. Equation (8) with $\lambda=1 / 2$ is often called the Homann differential equation. In the following we obtain a theorem concerning non-oscillatory behaviour of solutions of non-homogeneous equation associated with Equation (8), that is,

$$
y^{\prime \prime \prime}+y y^{\prime \prime}+\lambda\left[1-\left(y^{\prime}\right)^{2}\right]=f(t),
$$

where $f \in C([a, \infty), R)$ is such that $f(t) \geq 0$.

THEOREM 7. If $-1 \leq \lambda<0$ then all solutions of Equation (11) are non-oscillatory. If $\lambda>0$ and $\lim _{t \rightarrow \infty} f(t)=\infty$, then all solutions of Equation (11) are non-oscillatory. If $\lambda<-1$ and $\lim _{t \rightarrow \infty} f(t)=\infty$ then all solutions of Equation (11) whose first derivatives are bounded are non-oscillatory.

PROOF. The equation (11) can be written as

$$
\left(y^{\prime \prime}+y y^{\prime}\right)^{\prime}=(1+\lambda)\left(y^{\prime}\right)^{2}+f(t)-\lambda .
$$

In each case we see that the right-hand side of the above identity is positive for sufficiently large $t$. Then proceeding as in Theorem 1 we may show that all solutions of (11) are non-oscillatory. Hence the proof of the theorem is complete.

\section{EXAMPLES.}

(i) All solutions of

$$
y^{\prime \prime \prime}+y y^{\prime \prime}-\left[1-\left(y^{\prime}\right)^{2}\right]=6 t^{2}-1, \quad t \geq 1,
$$

are non-oscillatory. In particular, $y(t)=t^{2}$ is a non-oscillatory solution of the equation. 
(ii) The equation

$$
y^{\prime \prime \prime}+y y^{\prime \prime}+\left[1-\left(y^{\prime}\right)^{2}\right]=1+\frac{7}{t^{4}}, \quad t \geq 1,
$$

is non-oscillatory with a particular non-oscillatory solution $y(t)=-1 / t$.

(iii) The equation

$$
y^{\prime \prime \prime}+y y^{\prime \prime}+\left[1-\left(y^{\prime}\right)^{2}\right]=1+e^{t}, \quad t \geq 0,
$$

is non-oscillatory. In particular, $y(t)=e^{t}$ is a non-oscillatory solution of the equation.

\section{Relation between linearly independent solutions}

In this section we study the relation between three linearly independent solutions of $(\mathrm{E})$. Let $y_{1}(t), y_{2}(t)$ and $y_{3}(t)$ be solutions of (E) with initial conditions

$$
\begin{array}{lll}
y_{1}(a)=0 & y_{1}^{\prime}(a)=1 & y_{1}^{\prime \prime}(a)=0 \\
y_{2}(a)=1 & y_{2}^{\prime}(a)=0 & y_{2}^{\prime \prime}(a)=-q(a) / r(a) \\
y_{3}(a)=0 & y_{3}^{\prime}(a)=0 & y_{3}^{\prime \prime}(a)=1 / r(a)
\end{array}
$$

THEOREM 8. If $p(t) \leq 0, q(t) \leq 0$ and $q^{\prime}(t) \geq 0$, then $y_{1}(t)$ cannot meet $y_{2}(t)$ in the strip $\left[a, t_{1}\right)$, where $t_{1}$ is given by

$$
t_{1} \geq 1+a+p(a) \int_{a}^{t_{1}}\left(\int_{a}^{s} \frac{d u}{r(u)}\right) d s .
$$

PROOF. From Theorem 1 it follows that $y_{1}(t)$ and $y_{2}(t)$ are non-oscillatory. Successive integrations yield

$$
\begin{aligned}
y_{1}(t)=(t-a)+\int_{a}^{t} & \left(\int_{a}^{u}\left(\frac{1}{r(s)} \int_{a}^{s} f(\theta) d \theta\right) d s\right) d u \\
& -\int_{a}^{t}\left(\int_{a}^{u} \frac{1}{r(s)}(p(s)+q(s)) y_{1}(s) d s\right) d u \\
& +\int_{a}^{t}\left(\int_{a}^{u} \frac{1}{r(s)}\left(\int_{a}^{s} q^{\prime}(\theta) y_{1}(\theta) d \theta\right) d s\right) d u
\end{aligned}
$$

and

$$
\begin{aligned}
y_{2}(t)=1+p(a) \int_{a}^{t} & \left(\int_{a}^{s} \frac{d u}{r(u)}\right) d s+\int_{a}^{t}\left(\int_{a}^{u}\left(\frac{1}{r(s)} \int_{a}^{s} f(\theta) d \theta\right) d s\right) d u \\
& -\int_{a}^{t}\left(\int_{a}^{u} \frac{p(s)+q(s)}{r(s)} y_{2}(s) d s\right) d u \\
& +\int_{a}^{t}\left(\int_{a}^{u}\left(\frac{1}{r(s)} \int_{a}^{s} q^{\prime}(\theta) y_{2}(\theta) d \theta\right) d s\right) d u .
\end{aligned}
$$


If $t_{1}>a$ is the first point where $y_{1}(t)$ meets $y_{2}(t)$, then $y_{1}\left(t_{1}\right)=y_{2}\left(t_{1}\right)$ and $y_{1}(t)<y_{2}(t)$ for $t \in\left[a, t_{1}\right)$. Thus $y_{2}\left(t_{1}\right) \geq 1+p(a) \int_{a}^{t_{1}}\left(\int_{a}^{s} d u / r(u)\right) d s+y_{1}\left(t_{1}\right)-\left(t_{1}-a\right)$, that is,

$$
t_{1} \geq 1+a+p(a) \int_{a}^{t_{1}}\left(\int_{a}^{s} \frac{d u}{r(u)}\right) d s
$$

Hence the theorem is proved.

REMARK. The conclusion of Theorem 8 holds if

(i) $p(t) \geq 0, q(t) \leq 0$, such that $p(t)+q(t) \leq 0$ and $q^{\prime}(t) \geq 0$;

(ii) $p(t) \leq 0, q(t) \geq 0$ such that $p(t)+q(t) \leq 0$ and $q^{\prime}(t) \geq 0$

However, if $p(t) \geq 0, q(t) \geq 0$ and $q^{\prime}(t) \leq 0$, then $y_{1}(t)$ cannot meet $y_{2}(t)$ in the strip $\left[a, t_{1}\right)$, where $t_{1}$ is given by

$$
t_{1} \leq 1+a+p(a) \int_{a}^{t_{1}}\left(\int_{a}^{s} \frac{d u}{r(u)}\right) d s
$$

THEOREM 9. If $p(t) \leq 0, q(t) \leq 0$ and $q^{\prime}(t) \geq 0$, then $y_{3}(t)$ cannot meet $y_{1}(t)$ in the strip $\left(a, t_{1}\right)$, where $t_{1}$ is given by

$$
t_{1} \leq a+\int_{a}^{t_{1}}\left(\int_{a}^{s} \frac{d u}{r(u)}\right) d s
$$

and $y_{3}(t)$ cannot meet $y_{2}(t)$ in the strip $\left[a, t_{1}\right)$, where $t_{1}$ is given by

$$
1 \leq(1-p(a)) \int_{a}^{t_{1}}\left(\int_{a}^{s} \frac{d u}{r(u)}\right) d s
$$

The proof of this theorem is similar to that of Theorem 8 and hence is omitted.

REMARK. The conclusion of the above theorem remains true if

(i) $p(t) \geq 0, q(t) \leq 0$, such that $p(t)+q(t) \leq 0$ and $q^{\prime}(t) \geq 0$,

(ii) $p(t) \leq 0, q(t) \geq 0$ such that $p(t)+q(t) \leq 0$ and $q^{\prime}(t) \geq 0$.

\section{Acknowledgement}

I thank Dr S. K. Nayak for bringing the Blasius equation to my notice. I also thank the referee for his remarks which helped to improve the paper. 


\section{References}

[1] J. H. Barrett, 'Oscillation theory of ordinary linear differential equations', Adv. in Math. 3 (1969), 415-509.

[2] P. Hartman, Ordinary differential equations (Wiley, New York, 1964).

[3] G. Jayaraman, N. Padmanabhan and R. Mehrotra, 'Entry flow into a circular tube of slowly varying cross-section', in: Fluid Dynamics Research 1 (The Japan Society of Fluid Mechanics, 1986) pp. 131-144.

[4] N. Parhi, 'Nonoscillatory behaviour of solutions of nonhomogeneous third order differential equations', Applicable Anal. 12 (1981), 273-285.

[5] N. Parhi and S. Parhi, 'Qualitative behaviour of solutions of forced nonlinear third order differential equations', Riv. Mat. Univ. Parma (4) 13 (1987), 201-210.

[6] C. A. Swanson, Comparison and oscillation theory of linear differential equations (Academic Press, New York, 1968).

Berhampur University

Berhampur - 760007

India 